\title{
CORRESPONDENCE
}

\section{Questioning how different societies respond to crises}

In his Review of our book Questioning Collapse (Nature 463, 880-881; 2010), Jared Diamond alleges that it contains factual errors, which he uses to justify his devaluation of our emphasis on human resilience. In doing so, he shores up what we contend are his simplistic theses regarding societal 'collapse'. Given that our book critically examines two of Diamond's works - Guns, Germs, and Steel and Collapse: How Societies Choose to Fail or Succeed - we are surprised that Nature published this review without printing a fuller disclosure of the author's position in relation to our book.

We consider that there are errors in Diamond's review to which we wish to respond briefly. First, Niels Lynnerup, author of The Greenland Norse: A Biological-Anthropological Study (Meddelelser om Grønland, Man and Society, 1998), reiterated in a recent phone conversation with us that he knows of no evidence of starvation of the Greenland Norse. Second, as Chaco Canyon in New Mexico has never been sited in lush coniferous forest but in dry, scrub vegetation, wood for buildings was always imported from increasingly further away as nearby forests became exhausted. The search for wood was not the reason for Chaco's abandonment. Third, David Cahill's chapter shows that when Spaniards came to Peru, the Inka were engaged in a civil war as well as imperial expansion. Consequently, the Inka had many local enemies, some of whom allied with the Spaniards against the Inka. Fourth, Christopher Taylor's description of the culture and history of the Rwandan genocide refutes Diamond's 'Malthusian explanation' for this tragedy. And finally, we believe, based on considerable evidence, that the man named Yali whom Diamond declares to have met in
Papua New Guinea and the Yali discussed by Deborah Gewertz and Frederick Errington are the same person. Neither Diamond nor anyone else has produced evidence that would lead us to question this.

The point of Questioning Collapse is that everyone didn't "end up dead" in cases of 'collapse', but that many survived and some flourished under changed political and cultural circumstances. The conflation of profound societal change with the notion of biological extinction is a persistent error that runs through much 'collapse' scholarship. We believe that our book presents ample archaeological and historical data that contextualize how societies moved through periods of crisis. Our book is more than a critical evaluation of Diamond's views: it is about how we understand change in the past, how we grapple with the legacy of colonialism and with inequalities in the present, and how we can move forward productively and resiliently into the future.

Patricia A. McAnany Department of Anthropology, University of North Carolina, Chapel Hill, North Carolina 27599, USA

e-mail:mcanany@email.unc.edu Norman Yoffee Department of Near Eastern Studies and Department of Anthropology, University of Michigan, Ann Arbor, Michigan 48104, USA. The full list of 10 additional signatories is available online at http://dx.doi. org/10.038/464977a

\section{Students caught up in legal impasse at Mexican institute}

As graduate students from the nanoscience and nanotechnology group of the Institute for Scientific and Technological Research of San Luis Potosí (IPICYT), we wish to update you on events affecting us since the departure of Humberto and Mauricio Terrones (Nature 464, 148-149; 2010).

We have no wish to discredit the authorities of our institute, but we are concerned about the way in which they are handling our situation. It is evident that political and legal problems are interrupting our academic development.

The academic authorities at IPICYT have assured us that we would receive their complete support, so that we could continue with our thesis projects and dissertations. Most of our laboratory requirements have been met, and the authorities have agreed that we could choose our own advisers, whether from inside the institute or outside including the Terrones professors.

However, the current legal situation means that, if we did choose Humberto or Mauricio Terrones, they would be unable to supervise or examine us on IPICYT premises. And it is not clear whether the IPICYT authorities would actually recognize academic connections between students and the

Terrones professors.

In that case, we would need either to remain at the institute and terminate academic relations with the Terrones, or to continue our research and academic studies elsewhere. Neither option, however, would enable us to complete our thesis projects satisfactorily.

Aarón Morelos Gómez, Eduardo Gracia Espino, Juan Carlos García Gallegos and students of the Nanoscience and Nanotechnology Group, IPICYT, Camino a la Presa San José 2055, Col. Lomas 4a. Secc. SLP, CP78216, México e-mail: estudiantesnyn@gmail.com The full list of additional signatories is available online at http://dx.doi. org/10.038/464977b

\section{Weighing up the case for telescope site on La Palma}

The European Southern Observatory (ESO) Council will soon decide where to install the European Extremely Large Telescope (E-ELT). The currently recommended site is Armazones, a sierra near Paranal in Chile (Nature 464, 146; 2010). But there is also a strong case for considering the Roque de los Muchachos Observatory (ORM) on the Canary Island of La Palma.

This European site has the support of the European parliament and meets the astronomical requirements and logistical services necessary to make the operation cost-effective. For example, the E-ELT will need to use adaptive optics, which require a high-quality atmosphere in which the turbulent layers are as near to the telescope entrance as possible: the ORM's stable and predictable atmosphere makes it the best-quality site under consideration, as well as the most well studied.

Spain has offered the $€ 300$ million (US\$400 million) needed to implement the project. Siting the telescope in this European ultra-peripheral region would make it eligible for additional funding for its construction and operation.

At the meeting of the European Conference on Research Infrastructures held last month in Barcelona, it was stressed that all European research-infrastructure projects must minimize and optimize their construction and operational costs. These stipulations could be fulfilled by choosing the ORM option.

Another factor is the high seismicity of the Armazones region, which could affect the E-ELT's huge structure, multiple mirrors, complex instrumentation and adaptive optics. Additional security provisions could double the estimated costs.

European astronomy should not put all its eggs into one shaky basket, when there is an alternative secure site nearer home that offers equally good astronomical conditions.

Francisco Sanchez Instituto de Astrofísica de Canarias, Via Lactea s/n, La Laguna, Tenerife 38200, Canary Islands, Spain

Contributions may be submitted to correspondence@nature.com. 